\title{
Social Isolation and Loneliness During the COVID-19 Pandemic: Impact on Weight
}

\author{
Leslie J. Heinberg ${ }^{1}$ (1) $\cdot$ Kristine Steffen ${ }^{2}$ \\ Accepted: 22 June 2021 / Published online: 23 July 2021 \\ (C) The Author(s), under exclusive licence to Springer Science+Business Media, LLC, part of Springer Nature 2021
}

\begin{abstract}
Purpose of Review Social isolation and loneliness have long been identified as risk factors for poorer physical and mental health and increased mortality. These factors have also been shown to impact dietary behavior and physical activity which play a role in precipitating and maintaining obesity. Less is known about the impact of social isolation resulting from the COVID-19 pandemic in which social distancing is a major component of public health initiatives. This narrative review will examine the existing literature on the relationships between social isolation, loneliness, mental health, and weight as they relate to the COVID-19 pandemic.

Recent Findings Individuals with obesity are at very high risk for worsening course of COVID-19, hospitalization, and death. This population may also be more significantly impacted by the dietary and physical activity consequences resulting from lockdown, social distancing, and isolation.

Summary The pandemic has led to significant lifestyle disruptions. However, early studies have largely relied upon crosssectional studies or convenience samples. Future research will need to study the impact more rigorously, particularly among populations at greatest risk.
\end{abstract}

Keywords COVID-19 $\cdot$ Obesity $\cdot$ Social isolation $\cdot$ Loneliness $\cdot$ dietary behavior $\cdot$ physical activity

\section{Introduction to Social Isolation, Loneliness, and Weight}

The SARS-CoV-2 coronavirus originated in late 2019 in the Wuhan province of China. Over the next few months, it spread internationally and the outbreak of the disease resulting from the virus (COVID-19) was declared a pandemic by the World Health Organization on March 11, 2020. By the first week of February 2021, 26.4 million cases have been reported in the USA with 449,000 deaths [1]. The virus has been shown to disproportionately affect, older individuals, people of color, and individuals with chronic health conditions [2]. These

This article is part of the Topical Collection on Psychological Issues

Leslie J. Heinberg

heinbel@ccf.org

1 Cleveland Clinic Lerner College of Medicine, 9500 Euclid Ave./ M61, Cleveland, $\mathrm{OH} 44195$, USA

2 North Dakota State University, Sanford Center for Bio-Behavioral Research, Fargo, ND, USA factors correlate with obesity. However, independent of the aforementioned risks, patients with obesity are particularly vulnerable to more severe disease, hospitalization, need for intensive care, and mortality $[3,4 \cdot \bullet]$.

Once the pandemic had been declared, public health experts made a number of changes to mitigate the spread. Although initially thought to be temporary, physical and social distancing, telework, virtual classrooms, curfews, lockdowns, etc. have become a way of life and have had a significant impact on social interaction. Social distancing has entered our popular vernacular but comes from the Centers for Disease Control's recommendations to (1) Stay at least 6 feet away from other people; (2) To not gather in groups; and (3) Stay out of crowded places and avoid mass gatherings [5]. Social distancing is likely the safest way to avoid exposure to SARS-CoV-2, but an untoward side effect of these mitigation efforts is an increase in social isolation. Although the term "social distancing" is widely used, this is typically meant to convey "physical distancing" to alleviate the spread of the virus, as opposed to suggesting that social communication through telephone or virtual platforms be avoided. The following text will provide a narrative review of the impact that 
social isolation resulting from the pandemic - and the resultant loneliness - has had on weight with a particular focus on individuals with obesity. These impacts from COVID-19 have the potential to worsen noncommunicable diseases such as obesity $[6 \bullet \cdot$. In order to better understand this relationship, we will briefly review the effect of social isolation and then will review the impact of social distancing and isolation on the following domains which most likely moderate and/or mediate the relationship on weight: mental health, dietary intake, disordered eating, and physical activity. Literature that specifically examines populations with obesity will be included in each section although these studies are much more limited.

\section{Social Isolation}

COVID-19 has had a devastating impact on the economy, employment, health, and access to medical and mental health care. Public health and governmental efforts to reduce the spread of the virus have led to unprecedented social distancing initiatives. Social distancing is a set of actions, often including reductions in face-to-face contact that are taken in order to slow or stop the spread of disease. Social isolation has been defined objectively as a number of social contacts. Finally, loneliness is perceived social isolation. It generally refers to an individual's appraisal of the quality of social relationships. Although instrumental in mitigating the exponential replicability of the virus, social distancing initiatives have led to increased social isolation and decreased social and community supports [7]. Social connection is fundamental to human nature and failure, or an inability, to fulfill this need has an impact on physical and mental health [8•]. Meta-analyses demonstrate that individuals reporting social isolation have $29 \%$ higher mortality and those reporting perceived social isolation (i.e., loneliness) have a $23 \%$ higher mortality, even after controlling for other demographic and health risk factors [9]. Those with greater reported loneliness also demonstrate worse physical health and more chronic disease [8•, 10, 11]. This literature focuses on self-reported social contacts and isolation and/or on indices of social interaction. These findings may be helpful in guiding hypotheses about the impact of current public health efforts - in which social isolation is recommended and, in some jurisdictions, mandated by law. However, it is not known if the current social changes will have a similar impact.

\section{Mental Health Implications of COVID-19 and Social Isolation/Loneliness}

Awareness of the impact of COVID-19 on mental health has continued to increase since the start of the pandemic. Numerous articles and commentaries have focused on this issue and it is highly apparent in the clinical setting that mental health has deteriorated for some patients as a result of the stressors associated with the pandemic. Most of this work, however, has focused on the confluence of COVID-19 stressors rather than specifically on the impact of social isolation. Overall, it has been suggested that some individuals may be at particularly high risk of COVID-19 related psychosocial outcomes, including those who have compromised immune function, those who live in or receive care from congregate settings, people with preexisting medical, psychiatric, or substance use problems [12]. Health care professionals are also uniquely positioned to experience emotional distress associated with the pandemic due to personal risk of viral exposure to themselves and loved ones, long working hours, shortages of personal protective equipment, and facing ethically challenging decision-making concerning resource allocation [12]. A recent systematic review examined the direct neuropsychiatric and indirect effects on mental health associated with the pandemic from 43 studies in the literature [13*•]. Patients who had experienced COVID-19 had a high level of post-traumatic stress symptoms and a significantly higher level of depressive symptoms, and patients with pre-existing psychiatric conditions had worsening of their symptoms [13・•]. As previously noted, patients with obesity are more likely to have severe COVID-19 and be hospitalized [3, 4.•]. Thus, they may be particularly at risk of a variety of adverse physical and psychiatric problems as a result of COVID-19. This study also found that health care workers had increased rates of depression, anxiety, psychological distress, and poor sleep quality. Among the general population, psychological well-being was lower and depression and anxiety were higher after COVID19 began relative to before, and this difference did not appear to change over a four-week period of evaluation during the pandemic. Factors that were associated with increased risk for anxiety and depression among health care workers and the general population varied across studies, but included factors such as sociodemographics (e.g., living alone, student status, living in an urban area, female gender), current or past medical history, including psychiatric disorders and substance abuse, psychological and social factors (e.g., poor-self rated health, poor sleep quality, higher perceived stress, unsteady family income), and job-related factors (e.g., frontline worker, $>10$ years of working) $[13 \bullet \bullet$.

More specifically, social isolation and disruption in mental health care and supports has led to speculation of a potential mental health crisis including increased rates of suicide [7] with others predicting an additional 27,644 "deaths of despair" (suicide and drug/alcohol deaths) due to COVID-19 [14]. Reviews of past disease-related quarantines indicate increased distress (fear, boredom, frustration, and stigma) and psychiatric symptoms (depressed mood, irritability, posttraumatic stress disorder symptoms, insomnia, and anxiety [15]. A history of pre-quarantine psychiatric illness has been 
shown to be a predictor for greater psychiatric sequelae [16॰]. The potential for other adverse psychiatric outcomes is high particularly relating to depressive disorders $[17 \bullet \bullet]$. Since the onset of stay-at-home orders, an online survey of patients in a medical weight management/bariatric surgery program found $83.6 \%$ endorsed increasing depressive symptoms [17••]. There is also a concerning interactive effect between psychiatric illness, obesity, and health behaviors seen during the pandemic. Data from the UK demonstrate that $79 \%$ of subjects had a decline in one or more lifestyle behaviors that protect against weight gain; these declines were greater in those reporting high levels of stress while those with psychiatric illness and obesity were most likely to report declines [18]. Other international studies have demonstrated that persons with obesity may be at greater risk. For example, a Brazilian cross-sectional study found that women with overweight were more likely to experience sadness, anxiety, depression, and low self-esteem even after adjusting for a number of clinical and demographic factors including days of social isolation [19].

\section{Social Isolation, Loneliness, and Eating Behaviors/Disordered Eating}

A number of factors related to COVID-19 and the subsequent social isolation and lifestyle disruption may influence the way that individuals eat. Early data coming out of the last year does indicate that the social disruption has impacted eating behaviors. For example, in a representational national sample recruited through social media and paid advertisements, positive changes such as decreased dining out, fast food consumption and breakfast skipping, and increased cooking at home were noted $[6 \bullet \cdot$. However, this same study found a $43.5 \%$ increase in unhealthy snacking, sweets consumption, and sugarsweetened beverages. In examining participants with obesity, $33.4 \%$ endorsed weight gain during the pandemic (versus $20.5 \%$ of those with overweight and $24.7 \%$ of those in normal-weight categories [6••]. Similarly, in a UK-based study of eating behavior during the lockdown, higher BMI was associated with lower levels of physical activity, poorer diet quality, and greater frequency in overeating [20]. These relationships were more significant in participants with class II and above obesity versus those with class I [20]. In a recent survey of patients undergoing obesity treatment, $69.6 \%$ of patients reported greater difficulty in achieving weight loss goals with $61.2 \%$ endorsing more stress eating since the onset of the pandemic $[17 \bullet \cdot 0$. Finally, recent work is showing this link among patients with severe obesity or those who've received bariatric surgery. In a single-site study in the USA, bariatric surgery patients early in the epidemic reported high levels of loneliness $(36.2 \%)$ and decreased social support $(23.2 \%)$ while noting increased emotional distress and problematic eating behaviors (loss of control eating: $48.2 \%$ ) [20]. A Brazilian study reported that adherence to social distancing guidelines was related to more sedentary behavior and less physical activity [21] and an Italian study among individuals who had a history of bariatric surgery reported that $37 \%$ felt lonelier with $66 \%$ indicating an increase in hunger [22].

The social isolation and subsequent loneliness that has occurred in the last year may be impacting these alterations in eating behavior [23]. Previously, it has been hypothesized that appetite dysregulation may be a potential pathway through which social isolation may influence such untoward health outcomes [24]. In addition, social isolation may have a larger impact on other lifestyle behaviors such as dietary intake, particularly eating pathology. Social isolation has been associated with disordered eating in cross-sectional studies [25, 26]. Eating episodes and calories consumed tend to increase in times of emotional distress - particularly depression - boredom and loneliness [23, 27]. Negative affect and perceived social isolation have also been shown to be associated with greater binge eating in a daily diary study and social isolation is a unique predictor of disordered eating behavior [28]. Other work demonstrates that loneliness mediates the relationship between emotional dysregulation and bulimia and binge eating disorder [24]. Research has also demonstrated that simply thinking about comfort foods (which are often highly caloric) decreases feelings of loneliness [29]. The current food availability climate may exacerbate some of these previously demonstrated relationships. Food shortages, stay-at-home orders, and anxiety about shopping have led many to favor shelf-stable processed foods and to purchase larger quantities. As persons work from home and shelter in place, they have easy availability to these food purchases. This may lead vulnerable patients to engage in more binge and graze eating. Such alterations have been noted in a study of patients with eating disorders in the USA and the Netherlands. In this study, $49 \%$ of participants noted bingeing on food stockpiled for their families [30]. Across weight loss treatments, this may impact weight loss, lead to weight regain, and undermine health benefits that may have been achieved. In bariatric surgery, patients' failure to adhere to dietary and supplementation recommendations could cause acute symptoms (e.g., dumping) and undermine long-term outcomes. Concerns have also been noted in eating disorders populations as lifestyle disruption, social isolation, and constraints on physical activity may increase weight and shape concerns and increase disordered eating behavior [31].

This literature, however, has focused on social contact deficits and perceived loneliness. The impact of the recent mandated social isolation is less known but beginning to be evaluated. For example, loss of control eating, cognitive restriction, and emotional eating behaviors were noted to have increased during a national lockdown (within Turkey) in comparison to pre-lockdown behaviors [27]. Emotional eating also increased pre- to post-lockdown in Spain [32]. In an Italian 
study of patients from a multidisciplinary weight loss program, a post-lockdown increase in boredom, loneliness, eating, and unhealthy snacks/sweets was described [33].

Although individuals are less likely to go to bars or order alcohol in restaurants due to local and state restrictions, drinking behavior has been shown to increase during the earliest phase of the pandemic. In a cross-sectional online survey of almost 1000 adults, individuals reported consuming more drinks per day and a greater proportion exceeded recommended drinking limits in April 2020 in comparison to February 2020 [34•]. These differences were found across all sociodemographic sub-groups [34•]. Additionally, a Brazilian study found greater alcohol consumption in men with overweight compared to those with a normal BMI but this relationship was not evident in women [35]. Although concerning in itself, this increased intake may also lead to weight gain given the caloric increase as well as the potential disinhibition of eating behaviors.

\section{Stay-at-Home Orders, Social Isolation, Physical Activity, and Sedentary Behavior}

A further undesired consequence of stay-at-home orders and social distancing has been reduced opportunities for physical activity (PA), an increase in sedentary behavior, and propensity for systemic deconditioning and worsening chronic health conditions $[36,37]$. PA has been recommended as potentially protective during the crisis [36] and may help mitigate the physical and mental health consequences of the pandemic [38, 39]. However, work has also shown that reduced movement outside of the house - where presumably PA is more likely to occur-is associated with other conventional health behaviors [40]. Therefore, it may be difficult to encourage PA at the same time as encouraging social distancing that may benefit viral exposure.

In the previously described Flanagan et al. study (2021), leisure sedentary activities increased by almost $17 \mathrm{~min}$ on weekends and $21 \mathrm{~min}$ on weekdays. Physical activity levels also decreased across all BMI categories during the pandemic as did the intensity of PA [6••]. A cross-sectional study out of the UK demonstrated that a higher BMI was significantly associated with lower PA levels during lockdown [18]. Unfortunately, global smartphone data (187 countries; 455,404 users) show a $27.3 \%$ ( $23 \%$ within the USA) reduction in daily step counts [41••]. Additionally, patients undergoing obesity treatment self-reported reductions in physical activity $(47.9 \%)$ and intensity $(55.8 \%)$ [17••].

\section{Observations on Implications in the Future}

Given the recent nature of the pandemic and the need for a rapid increase in knowledge, much of the literature has relied upon cross-sectional analyses, convenience samples, and recruitment through social media. This has implications for assessing causality and for the representativeness of the findings. Future work needs to longitudinally evaluate the impact of the COVID-19 pandemic and its lifestyle disruptions on the population as well as higher risk, vulnerable populations such as those with obesity. The COVID-19 pandemic is projected to persist well into 2021 and, even after the delivery of effective vaccines, social distancing remains a primary public health intervention. Furthermore, its biological, behavioral, psychological, and socioeconomic effects are anticipated to be felt for many subsequent years. Therefore, it is critical to understand how mental health, dietary behavior, disordered eating, and physical and sedentary activity will be impacted. Through multiple mechanisms, the COVID-19 pandemic has the potential to undermine weight loss treatment efficacy and impact patients who may be at the highest risk of severe complications and death. Such knowledge will help providers effectively mitigate: additional stressors, poorer weight outcomes, reemergence of comorbidities, psychological complications, undermined surgical efficiency, and greater healthcare costs and resource use.

\section{Conclusions}

The coronavirus SARS-CoV-2 (COVID-19) has caused significant lifestyle disruption. Stay-at-home orders, social isolation, and loss of routines and supports have resulted in largely universal vulnerability to overeating, reduced physical activity level, and sedentary behavior. Additionally, stress related to COVID-19 and social isolation may place individuals at amplified risk for worsening depression symptoms and unhealthy coping strategies such as increased alcohol consumption and binge eating. Patients with obesity, who are at significantly high risk for severe symptoms, hospitalizations, ICU stays, and death, may be at increased risk for negative disruption in health behaviors and future research is needed to better clarify risk for this population

\section{References}

Papers of particular interest, published recently, have been highlighted as:

- Of importance

- Of major importance

1. Centers for Disease Control and Prevention. United States COVID19 cases and deaths by state. https://covid.cdc.gov/covid-data- 
tracker/\#cases_casesper100klast7days. Accessibility verified February 5, 2021.

2. Centers for Disease Control and Prevention. People at increased risk and other people who need to take extra precautions. Accessibility verified February 5, 2021.

3. Tartof SY, Qian L, Hong V, Wei R, Nadjafi RF, Fischer H, et al. Obesity and mortality among patients diagnosed with COVID-19: Results From an Integrated Health Care Organization. Ann Intern Med. 2020;173(10):773-81. https://doi.org/10.7326/M20-3742.

4.• Tamara A, Tahapary DL, Tamara A, et al. Obesity as a predictor for a poor prognosis of COVID-19: a systematic review. Diabetes Metab Syndr. 2020;14(4):655-9 Individuals with obesity are at significantly higher risk for poor outcomes of COVID-19.

5. Centers for Disease Control and Prevention. Social distancing: Keep a safe distance to slow the spread. https://www.cdc.gov/ coronavirus/2019-ncov/prevent-getting-sick/social-distancing. html. Accessibility verified February 5, 2021.

6.• Flanagan EW, Beyl RA, Fearnbach SN, Altazan AD, Martin CK, Redman LM. The impact of COVID-19 stay-at-home orders on health behaviors in adults. Obesity. 2021;29(2):438-45 Examination of health behaviors in a large US sample.

7. Reger MA, Stanley IH, Joiner TE. Suicide mortality and coronavirus disease 2019 - a perfect storm? JAMA Psychiatry. 2020; online ahead of print.

8. Leigh-Hunt N, Bagguley D, Bash K, et al. An overview of systematic reviews on the public health consequences of social isolation and loneliness. Public Health. 2017;152:157-71 A review of reviews on the impact of social isolation and loneliness.

9. Holt-Lunstad J, Smith TB, Layton JB. Social relationships and mortality risk: a meta-analytic review. PLoS Med. 2010;7: e1000316.

10. Thurston RC, Kubzansky LD. Women, loneliness, and incident coronary heart disease. Psychosom Med. 2009;71(8):836.

11. Thurston RC, Kubzansky LD. N Engl J Med. 2020;383(6):510-2.

12. Pfefferbaum B, North CS, Pfefferbaum B, et al. Mental health and the Covid-19 pandemic. N Engl J Med. 2020;383(6):510-2. https:// doi.org/10.1056/NEJMp2008017.

13.• Vindegaard N, Benros ME, Vindegaard N, et al. COVID-19 pandemic and mental health consequences: systematic review of the current evidence. Brain Behav Immun. 2020;89:531-42. https:// doi.org/10.1016/j.bbi.2020.05.048 An early systematic review of the impact of mental health from COVID-19.

14. Petterson S, Westfall JM, Miller BF. Projected deaths of despair from COVID-19. https://digitalcommons.psjhealth.org/ publications $/ 3054$

15. Brooks SK, Webster RK, Smith LE, et al. The psychological impact of quarantine and how to reduce it: rapid review of the evidence. Lancet. 2020;395(10227):912-20. https://doi.org/10.1016/S01406736(20)30460-8.

16. Jeong H, Yim HW, Song YJ, et al. Mental health status of people isolated due to Middle East Respiratory Syndrome. Epidemiol Health. 2016;38:e2016048. https://doi.org/10.4178/epih.e2016048 Impact on mental health from a prior pandemic.

17.• Almandoz JP, Xie L, Schellinger JN, et al. Impact of COVID-19 stay-at-home orders on weight-related behaviors among patients with obesity. Clin Obes. 2020:e12386. https://doi.org/10.1111/ cob.12386 Examination of weight-related behavior change in obesity treatment-seeking individuals.

18. Robinson E, Gillespie SM, Jones A. Weight-related lifestyle behaviors and the COVID-19 crisis: An online survey study of UK adults during social lockdown. Obes Sci Pract. 2020;6:735-740. https:// doi.org/10.1002/osp4.442.

19. Lofrano-Prado MC, Luiz do Prado W, Botero JP, et al, The same storm but not the same boat: Effects of COVID-19 stay-at-home order on mental health in individuals with overweight. Clin Obes. 2021;11:e12425. https://doi.org/10.1111/cob.12425.
20. Athanasiadis DL, Hernandez E, Hilgendorf W, et al. How are baritric patients coping during the coronavirus disease 2019 *COVID-19 pandemic? Analysis of factors known to cause weight regain among postoperative bariatric patients. Surg Obes Relat Dis. 2021;17(4):756-64.

21. Nicoletti CF, Esteves GP, Genario R, et al. Nutritional inadequacies among post-bariatric patients during COVID-19 quarantine in Sao Paulo. Brazil Obes Surg. 2021;5:2330-4.

22. Sisto A, Vicinanza F, Tuccinardi D, Watanabe M, Gallo IF, D'Alessio R, et al. The psychological impact of COVID-19 pandemic on patients included in a bariatric surgery program. Eat Weight Disord. 2020; Epub ahead of print.

23. Jaremka LM, Fagundes CP, Peng J, Belury MA, Andridge RR, Malarkey WB, et al. Loneliness predicts postprandial ghrelin and hunger in women. Horm Behav. 2015;70:57-63. https://doi.org/10. 1016/j.yhbeh.2015.01.011.

24. Southward MW, Christensen KA, Fettich KC, Weissman J, Berona $\mathrm{J}$, Chen EY. Loneliness mediates the relationship between emotion dysregulation and bulimia nervosa/binge eating disorder psychopathology in a clinical sample. Eat Weight Disord. 2014;19(4):50913.

25. Ray J Implicit and Explicit Social Isolation in Eating Disordered Individuals. Thesis (unpublished). Bowling Green State University: 2012.

26. Waller G, Dickson C, Ohanian V. Cognitive content in bulimic disorders: core beliefs and eating attitudes. Eat Behav. 2002;3(2): 171-8.

27. Elmacioğlu F, Emiroğlu E, Ülker MT, Kircali BÖ, Oruc S Evaluation of nutritional behavior related to COVID-19. Pub Health Nutr. 2020;24(3):512-518. https://doi.org/10.1017/ S1368980020004140.

28. Mason TB, Heron KE, Braitman AL, Lewis RJ. A daily diary study of perceived social isolation, dietary restraint, and negative affect in binge eating. Appetite. 2016;97:94-100.

29. Troisi JD, Gabriel S. Chicken soup really is good for the soul: "comfort food" fulfills the need to belong. Psychol Sci. 2011;22: $747-53$.

30. Termorshuizen JD, Watson HJ, Thornton LM, Borg S, Flatt RE, MacDermod CM, et al. Early impact of COVID-19 on individuals with self-reported eating disorders: a survey of $\sim 1000$ individuals in the United States and the Netherlands. Int J Eat Dis. 2020;53:1780 90.

31. Rodgers RF, Lombardo C, Cerolini S, Franko DL, Omori M, Fuller-Tyszkiewicz M, et al. The impact of the COVID-19 pandemic on eating disorder risk and symptoms. Int J Eat Disord. 2020;53: 1166-70.

32. López-Moreno M, Iglesias López MT, Miguel M, Garcés-Rimón. Physical and psychological effects related to food habits and lifestyle changes derived from COVID-19 home confinement in the Spanish population. Nutrients. 2020;12:3445.

33. Pellegrini M, Ponzo V, Rosato R, Scumaci E, Goitre I, Benso A, et al. Changes in weight and nutritional habits in adults with obesity during the "lockdown" period caused by the COVID-19 virus emergency. Nutrients. 2020;12:2016.

34. Barbosa C, Cowell AJ, Dowd WN. Alcohol consumption in response to the COVID-19 pandemic in the United States. J Addict Med. 2020. https://doi.org/10.1097/adm.0000000000000767 Examination of increased alcohol use during the pandemic

35. Lofrano-Prado MC, Luiz do Prado W, Botero JP, et al. The same storm but not the same boat: effects of COVID-19 stay-at-home order on mental health of individuals with overweight. Clin Obes. 2020; epub ahead of print.

36. Carter SJ, Baranauskas MN, Fly AD. Considerations for obesity, vitamin D, and physical activity amid the COVID-19 pandemic. Obesity (Silver Spring). 2020;28:1176-7. https://doi.org/10.1002/ oby. 22838 . 
37. Chen P, Mao L, Nassis GP, Harmer P, Ainsworth BE, Li F. Coronavirus disease (COVID-19): the need to maintain regular physical activity while taking precautions. J Sport Health Sci. 2020;9(2):103-4. https://doi.org/10.1016/j.jshs.2020.02.001.

38. Burtscher J, Burtscher M, Millet GP. (Indoor) isolation, stress and physical inactivity: vicious circles accelerated by Covid-19? Scand J Med Sci Sports. 2020;30:1544-1545. https://doi.org/10.1111/ sms. 13706

39. Jimenez-Pavon D, Carbonell-Baeza A, Lavie CJ. Physical exercise as therapy to fight against the mental and physical consequences of COVID-19 quarantine: special focus in older people. Prog Cardiovasc Dis. 2020;63:386-388. https://doi.org/10.1016/j.pcad. 2020.03.009
40. Bourassa KJ, Sbarra DA, Caspi A, Moffitt TE. Social distancing as a health behavior: county-level movement in the United States during the COVID-19 pandemic is associated with conventional health behaviors. Ann Behav Med. 2020;54:548-56.

41.• Tison GH, Avram R, Kuhar P, et al. Worldwide effect of COVID19 on physical activity: a descriptive study. Ann Intern Med. 2020. https://doi.org/10.7326/M20-2665 Impact on daily step-count following pandemic lockdowns across the globe.

Publisher's Note Springer Nature remains neutral with regard to jurisdictional claims in published maps and institutional affiliations. 